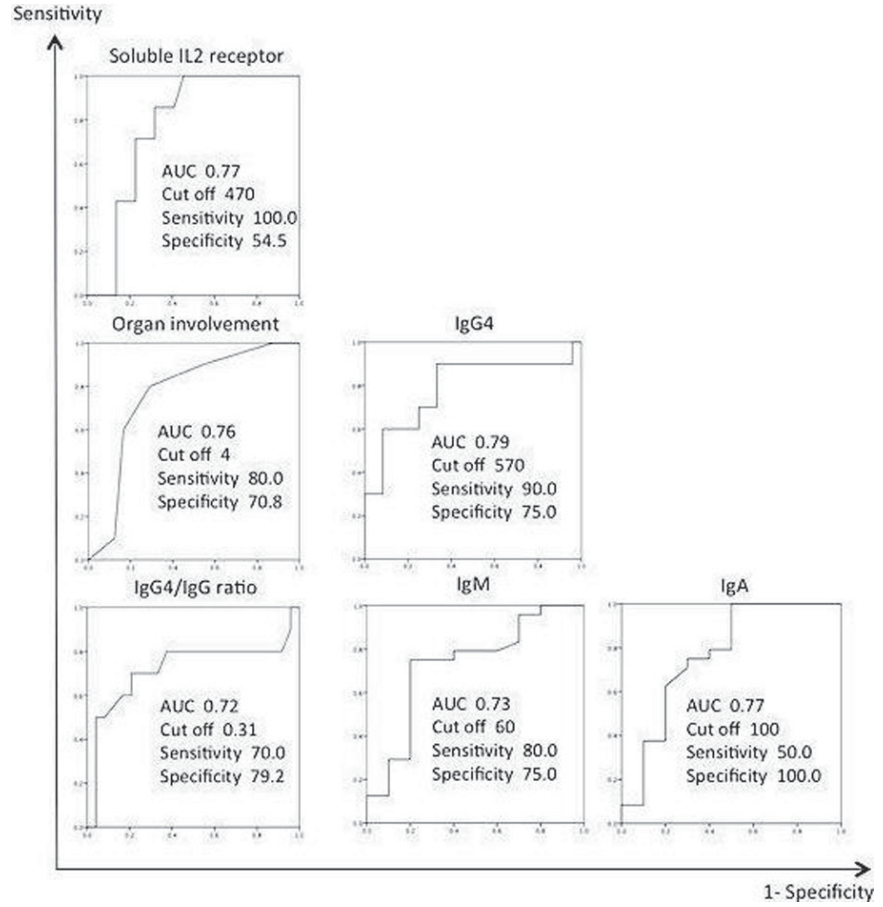

Figure 1

the higher likelihood of disease relapse following GCs therapy in patients with IgG4-RD.

References:

[1] Brito-Zerón P, Kostov B, Bosch X, Acar-Denizli N, Ramos-Casals M, Stone JH. Therapeutic approach to IgG4-related disease: A systematic review. Medicine (Baltimore). 2016;95:e4002.

Acknowledgements: We sincerely thank all the physicians and others caring for the patients enrolled in this study.

Disclosure of Interest: None declared

DOI: 10.1136/annrheumdis-2017-eular.4190

\section{FRI0592 CLINICAL, ANALYTICAL AND RADIOLOGICAL CHARACTERISTICS IN A COHORT OF PATIENTS WITH SARCOIDOSIS}

A. Ruiz Román ${ }^{1}$, C. Aguilera $\operatorname{Cros}^{1}$, M. Arcila Durán ${ }^{1}$, M. León Luque ${ }^{1}$, M. Lisbona Muñoz ${ }^{1}$, J.P. Sanchez Serrano ${ }^{2}$, J.A. Rodriguez Portal ${ }^{3}$

J. Povedano Gomez ${ }^{1} .{ }^{1}$ Rheumatology, Hospital Virgen del Rocio, Sevilla;

${ }^{2}$ Breeding and Genetics, Irta, Caldas de Montbui; ${ }^{3}$ Pneumology, Hospital Virgen del Rocio, Sevilla, Spain

Background: Sarcoidosis is a systemic granulomatous disease, frequently affecting lungs, eyes and skin, although it may damage other organs, among them the musculoskeletal system

Objectives: To describe the clinical characteristics and radiological pattern in a cohort with predominantly pulmonary sarcoidosis, and to determine the relationship between the levels of angiotensin converting enzyme (ACE), pulmonary radiological stage and sarcoidosis course (chronification or remission)

Methods: Data from 2328 patients in an Interstitial Lung Diseases consultation during the first half of 2016 were analyzed. Out of these, 50 had sarcoidosis. The delay in the diagnosis of sarcoidosis was defined as the difference in years between the diagnostic suspicion and diagnosis of sarcoidosis.

Chi square tests were used, assuming an error of the first species not higher than 0.05 , in order to: 1 . Study the association between angiotensin converting enzyme (ACE) levels and binary variables (extrapulmonary symptoms, radiological stage and evolution of S) 2. Determine the association between evolution, the radiological stage and the presence of extrapulmonary symptoms.

Results: We included 29 (58\%) women and 21 (42\%) men, (mean age of $44 \pm 11.7$ years). Initial diagnosis: $88 \%$ S, $8 \%$ lymphoma and $4 \%$ tuberculosis. Of the 44 diagnosed cases of S, 24 were on the first visit, 11 the following year and 1 seven years later. Of the 4 lymphomas, 2 were diagnosed of $S$ that same year and the other 2 were diagnosed the following year. Of the 2 tuberculosis, one was diagnosed of $S$ in one year and the other at 4 years.

The most frequent extrapulmonary manifestations were cutaneous $24 \%$, followed by the articular, cardiac and ocular in $10 \%$, neurological $8 \%$ and renal $4 \%$. In $6 \%$ of patients, the first clinical manifestation of the disease was bilateral arthritis of the ankles, The ACE title is increased in $62 \%$ of patients, normal in $34 \%$. The mean and standard deviation of the title of patients with an increased ACE value was 150.5 and $53.4 \mathrm{IU} / \mathrm{L}$, respectively. In all patients, x-ray and high resolution tomography were performed, with stage 2 being the most frequent $(44 \%)$, followed by $3(20 \%), 0$ and $1(14 \%)$ and $4(8 \%)$. Histological confirmation was obtained by transbronchial biopsy $(66 \%)$, cutaneous $(12 \%)$ or lymph node biopsy $(12 \%)$ in $90 \%$ of the patients. $90 \%$ of patients have been treated with oral glucocorticoids and $42 \%$ associate immunosuppressive therapy.

The ACE levels showed no statistical association with any of the variables studied, although a very clear association $(p=0.04754)$ was observed between the course of the disease and the presence of extrapulmonary symptoms: from the 25 patients without extrapulmonary symptoms, only in 35\% of cases the process become chronic.

Conclusions: Our results, in general, coincide with what is published in the literature. In our cohort, initial diagnosis of $S$ was relatively high $(28 / 50=56 \%)$, while misdiagnosis was relatively low $(6 / 50=12 \%)$. The level of ACE does not seem to be clearly associated with the presence of extrapulmonary symptoms, nor with the course of S. However, the presence of extra-pulmonary symptoms seems to lead to a chronification

Disclosure of Interest: None declared

DOI: 10.1136/annrheumdis-2017-eular.5480

\section{FRI0593 ASSOCIATION OF THYMOMA WITH AUTOIMMUNE DISEASES IN A SERIES OF 83 CASES}

A. González García ${ }^{1}$, W.A. Sifuentes Giraldo ${ }^{2}$, J.L. Morell Hita ${ }^{2}$, J.L. Patier de la Peña ${ }^{1}$. IInternal Medicine; ${ }^{2}$ Rheumatology, Ramon y Cajal University Hospital, Madrid, Spain

Background: Thymoma is the most common neoplasm originated from the thymus gland and accounting for $50 \%$ of anterior mediastinal tumors. Within its clinical manifestations are included the loss of self-tolerance and the development of autoimmunity.

Objectives: To study the frequency of autoimmune diseases (AD) in patients with thymoma and to describe their clinical characteristics and outcome.

Methods: We performed a retrospective observational study of a cohort of patients diagnosed with thymoma and followed-up in our center between January 1985 and September 2016. The variables evaluated included demographics, thymoma characteristics, clinical and analytical manifestations of autoimmunity, treatment and outcome.

Results: A total of 83 patients were included, $56.6 \%$ of them women, with a mean age at diagnosis of the thymoma of $58.4 \pm 15.8$ years (range: $16-94$ ), 31.3\% of which corresponded to type I The classification of Masaoka, $39.1 \%$ to II, $17.2 \%$ to III and $10.9 \%$ to IV. There were one or more AD associated in 41 cases $(49.4 \%)$. The most frequent diagnoses were myasthenia gravis (19), systemic lupus erythematosus (SLE) (4), subacute cutaneous lupus erythematosus (1), Sjögren's syndrome (1), rheumatoid arthritis (1), spondyloarthritis (1), sarcoidosis (1), hemolytic anemia (2), pernicious anemia (1), aplastic anemia (1), cutaneous limited systemic sclerosis (1), urticaria-vasculitis, erythroblastopenia (1), recurrent pericarditis (1), thyroid disease (2) and lichen planus (1). The diagnosis of AD preceded to thymoma in $38.2 \%$ of cases and was later in the remaining cases. In 4 cases there was also a concomitant primary immunodeficiency (variable common immunodeficiency 3, CD4 immunodeficiency 1). The most frequently identified autoantibodies were anti-acetylcholine receptor $(14 / 41,34.1 \%)$, anti-striated muscle $(3 / 41,7.3 \%)$, ANA $(11 / 41,26.8 \%),(3 / 41,7.3 \%)$, rheumatoid factor $(3 / 41$, $7.3 \%)$, anti-thyroid $(3 / 41,7.3 \%)$, antiphospholipids $(2 / 41,9 \%)$ and anticentromere $(1 / 41,2.4 \%)$. In the comparison of patients with and without associated AD, no significant differences were found regarding age, sex or Masaoka classification. There were 6 deaths, 4 in group with associated $A D$ and 2 in the group without $A D$, but without significant difference $(p=0.3797)$.

Conclusions: In the analyzed population of patients with thymoma of our center, almost half of them developed $A D$, which in a major group preceded the diagnosis of neoplasia. The spectrum of autoimmunity associated with thymoma was quite broad, including organ-specific AD such as myasthenia gravis (which is most frequently described in the literature) and autoimmune cytopenias, but also to systemic $A D$, the most common being SLE. The autoimmunity study should be included in the assessment of the patient with thymoma as it could contribute to the early diagnosis of associated AD.

Disclosure of Interest: None declared

DOI: 10.1136/annrheumdis-2017-eular.6992

\section{FRI0594 A CASE OF MOSAICISM IN TNF ASSOCIATED PERIODIC SYNDROME (TRAPS)}

A. Kontzias ${ }^{1}$, C. Calabrese ${ }^{1}$, Y.-W. Cheng ${ }^{2} .{ }^{1}$ Rheumatology and Immunology;

${ }^{2}$ Laboratory Medicine, Cleveland Clinic, Cleveland, United States

Background: Tumor necrosis factor receptor (TNFR)-associated periodic syndrome (TRAPS) is an autosomal-dominant disease caused by gain-of-function mutations in the TNFRSF1A gene, which encodes the 55-kd TNFR type I (TNFRI) protein. Mosaicism has been recently idenitfied in a single patient. ${ }^{1} \mathrm{~A} 60$ year old male presented with a 6 year history of intermittent fever as high as 103.5, lasting 3-4 weeks with associated peritoneal symptoms, arthralgias, myalgias, lymphadenopathy, bilateral episcleritis, erythematous rash in his torso. Prednisone up to $60 \mathrm{mg}$ daily only partially alleviated his symptoms and colchicine was ineffective. Objectives: To explore the role of mosaicism in a patient with adult onset TRAPS phenotype. 